\title{
Algebraic Multilevel Methods for Edge Elements
}

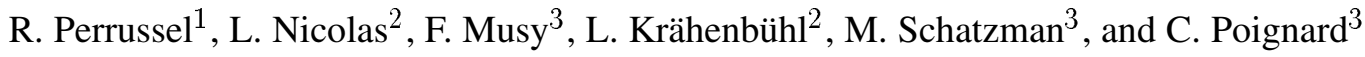 \\ ${ }^{1}$ Institut Camille Jordan (ICJ) and CEGELY, Ecole Centrale de Lyon 69134 Ecully Cedex, France \\ ${ }^{2}$ CEGELY, Ecole Centrale de Lyon 69134 Ecully Cedex, France \\ ${ }^{3}$ ICJ, Ecole Centrale de Lyon 69134 Ecully Cedex, France
}

\begin{abstract}
An algebraic multilevel method is proposed for the resolution of linear systems coming from an edge-element discretization of EM models. Graph-flow problems are introduced to ensure a natural compatibility condition linking nodal and edge interlevel transfer operators. The efficiency of our method is compared to classical solvers on two-dimensional and three-dimensional eddy current problems.
\end{abstract}

Index Terms-Algebraic multigrid methods, edge elements.

\section{INTRODUCTION}

$\mathbf{T}$ HE finite-element discretization of partial differential equations (PDEs) leads to large sparse linear systems. This is often the most time-consuming part of the finite-element computations. This part can be optimized in view of specific applications. The multilevel approach, also called multigrid, consists of considering the linear system at different mesh scales, which can substantially reduce the computational time. Although the multilevel concept is relatively generic, the practical components are narrowly linked to PDE, finite-element, and mesh properties. In this paper, we deal with EM models discretized by the lowest-order edge elements on an unstructured mesh, i.e., without a hierarchy of nested grids.

The starting point is the construction of coarse-nodal and coarse-edge functions which satisfy a natural compatibility relation: The gradient of coarse-nodal functions are linear combinations of coarse-edge functions. This relation is introduced as a constraint in an energy-minimization problem for constructing coarse bases. By linking the compatibility relation to graph-flow problems, the minimization problem can be reduced to a linear system. The efficiency of our approach is compared to classical solvers on two-dimensional (2-D) and three-dimensional (3-D) eddy current problems.

\section{FORMULATION}

The following problem has to be solved on a domain $\Omega$

$$
\left\{\begin{array}{l}
\text { Find } \mathbf{E} \in V_{h} \text { such that : } a\left(\mathbf{E}, \mathbf{E}^{\prime}\right)=F\left(\mathbf{E}^{\prime}\right), \forall \mathbf{E}^{\prime} \in V_{0} \\
\text { with } a\left(\mathbf{E}, \mathbf{E}^{\prime}\right)=\int_{\Omega} \delta \text { curl } \mathbf{E} \cdot \operatorname{curl} \mathbf{E}^{\prime}+\int_{\Omega} \alpha \mathbf{E} \cdot \mathbf{E}^{\prime} .
\end{array}\right.
$$

$V_{0}$ is an edge element subspace of $\mathbf{H}(\operatorname{curl}, \Omega), V_{h}$ is the affine space taking into account essential boundary conditions, $F$ is a source term, and $\delta$ and $\alpha$ are strictly positive functions. This formulation includes many static and transient EM models. It leads to solve the linear system

$$
A x=b .
$$

where the components of $x$ are the coefficients of the solution in the edge-element basis.

Digital Object Identifier 10.1109/TMAG.2006.871604

\section{Algebraic Multilevel Method}

\section{A. A Simple Two-Level Case}

Suppose that two levels of discretization are known for the problem under consideration. For instance, one could have discretizations on two nested meshes $T_{H}$ and $T_{h}$ where the subscript $h$ is used for the fine level and $H$ for the coarse level. Thus, $A_{h}, x_{h}$, and $b_{h}$ are, respectively, the matrix, the solution vector and the right-hand side on the fine level. A prolongation matrix $P$ transfers information from the coarse to the fine level. The transposed matrix $P^{T}$ is called restriction.

Two complementary steps are needed for the two-level algorithm [1, Section 1.5].

1) Smoothing. The "oscillating" part of the error is damped by a linear iteration called smoother $S$ (Gauss-Seidel type methods are often used) $x_{h} \leftarrow S\left(A_{h}, x_{h}, b_{h}\right)$. The new residual $r_{h}=A_{h} x_{h}-b_{h}$ is then transferred to the coarse level: $r_{H} \leftarrow P^{T} r_{h}$.

2) Correction. The "smooth" part of the error is computed on the coarse level $\theta_{H} \leftarrow A_{H}^{-1} r_{H}$ and is prolongated to the fine level as a correction: $x_{h} \leftarrow x_{h}-P \theta_{H}$.

The method is iterative and the two steps are repeated until the norm of the residual $\left\|r_{h}\right\|$ is sufficiently small. More complex variants are possible, using for instance pre- and postsmoothing. Replacing the correction step by a two-level method leads to the recursively defined multilevel method.

\section{B. Algebraic Multilevel}

Using a hierarchy of nested grids is the straightest way to implement multilevel techniques. Nevertheless, in some applications, only information at the fine level is available for building coarse levels; this is the case for unstructured meshes. Algebraic strategies must then be followed.

The main task is to define a coarse basis or equivalently the prolongation matrix $P$. For the recursive application of the multilevel method, the coarse matrix is assembled by the Galerkin product $A_{H}=P^{T} A_{h} P$.

\section{Edge Element Features}

Hiptmair [2] and Arnold et al. [3] have proposed appropriate smoothers for edge elements, specifically dealing with the kernel of the curl operator. For algebraic multilevel methods, 
the construction of the prolongation matrix must also be considered. Due to relations between first-order conforming nodal elements and lowest-order edge elements [4], an important compatibility relation must be ensured

$$
\operatorname{grad} \phi_{n}^{H}=\sum_{e=1}^{E^{H}} G_{\mathrm{en}}^{H} \lambda_{e}^{H}, \quad \forall n \in\left\{1, \ldots, N^{H}\right\}
$$

where $\left(\phi_{n}^{H}\right)_{n=1 \ldots N^{H}}$ is the coarse-nodal basis, $\left(\lambda_{e}^{H}\right)_{e=1 \ldots E^{H}}$ is the coarse-edge basis, and $G^{H}$ is the discrete analog of the gradient operator on the coarse level. The matrix $G^{H}$ can be viewed as the node-edge incidence matrix of an oriented coarse graph $\mathcal{S}^{H}$ defined by the relations

$$
\begin{aligned}
& e \text { index of } \\
& \text { an edge in } \mathcal{S}^{H}
\end{aligned} \Leftrightarrow G_{\mathrm{en}}^{H}= \begin{cases}-1, & \text { if node } n \text { is the origin } \\
+1, & \text { if node } n \text { is the end } \\
0, & \text { otherwise. }\end{cases}
$$

Enforcing (3) is a main issue for algebraic multilevel methods, as it was first highlighted by Reitzinger and Schöberl [5]. The compatibility condition was also used by Bochev et al. in [6], [7].

Edge and nodal coarse bases are constructed so as to satisfy the inclusion of finite-element spaces, the "coarse" being included in the "fine," which is expressed by the following algebraic relations:

$$
\begin{aligned}
& \phi_{n}^{H}=\sum_{p=1}^{N^{h}} P_{p n}^{\mathrm{nod}} \phi_{p}^{h}, \quad \forall n \in\left\{1, \ldots, N^{H}\right\} \\
& \lambda_{e}^{H}=\sum_{i=1}^{E^{h}} P_{\mathrm{ie}}^{\mathrm{edg}} \lambda_{i}^{h}, \quad \forall e \in\left\{1, \ldots, E^{H}\right\} .
\end{aligned}
$$

The matrices $P^{\text {nod }}$ and $P^{\text {edg }}$ are, respectively, the nodal and edge prolongation matrices which have to be constructed.

The analog of relation (3) is also assumed to be satisfied at the fine level

$$
\operatorname{grad} \phi_{p}^{h}=\sum_{i=1}^{E^{h}} G_{i p}^{h} \lambda_{i}^{h}, \quad \forall p \in\left\{1, \ldots, N^{h}\right\} .
$$

From relations (5) and (6), the compatibility condition (3) is algebraically written

$$
P^{\text {edg }} G^{H}=G^{h} P^{\text {nod }} .
$$

\section{NEW APPROACH}

In order to compute an efficient coarse basis verifying the compatibility relation, the "energy-minimizing coarse basis" concept from [8] is applied. First, a decomposition of the domain into overlapping subdomains is introduced $\Omega=\cup_{n=1}^{N^{H}} \Omega_{n}$. The support of the coarse nodal function $\phi_{n}^{H}$ is enforced to be included in $\Omega_{n}$, by setting equal to zero values in the $n$th column of $P^{\text {nod }}$.

Moreover, in order to ensure that the constant functions belong to the coarse nodal space, the sum of each row of $P^{\text {nod }}$ is enforced to be equal to one.
We compute the prolongation matrix $P^{\text {nod }}$ by usual techniques, for example, by smoothed aggregation [9].

Then, we define a node-edge incidence matrix $G^{H}$ or, equivalently, an oriented coarse graph such that for any coarse edge $e$, whose extremities are nodes $n$ and $m, \Omega_{n}$ and $\Omega_{m}$ intersect. Thus, for each coarse edge $e$, we introduce the subdomain $\mathcal{U}_{e}=\Omega_{n} \cap \Omega_{m}$ and the coarse edge function $\lambda_{e}^{H}$ whose support is enforced to be in $\overline{\mathcal{U}}_{e}$, by setting equal to zero values in the $e$ th row of $P^{\text {edg }}$.

Then, we solve the following optimization problem:

$$
\left\{\begin{array}{l}
\text { To find } P^{\text {edg }} \text { minimizing } \sum_{e=1}^{E^{H}} \beta_{e}^{T} K_{e} \beta_{e} \\
\text { under the constraint }(7) .
\end{array}\right.
$$

Here, $\beta_{e}$ is the vector where the nonzero values of the eth column of $P^{\text {edg }}$ are gathered, and $K_{e}$ is a symmetric positive definite matrix. More precisely, $K_{e}$ may be defined by the discretization of the form $a$ from (1) on $\mathcal{U}_{e}$, but variants can also be introduced to minimize the overhead of computing the solution of (8).

\section{SOLVING The Optimization Problem}

\section{A. Main Concept}

For each fine egde index $i$ such that a coarse edge of index $e$ exists with the coefficient $P_{i e}^{\text {edg }}$ not enforced to vanish, we introduce a matrix $G^{\mathrm{H}, \mathrm{i}}$. This matrix is the node-edge incidence matrix of the subgraph $\mathcal{S}^{\mathrm{H}, \mathrm{i}}$ obtained from the coarse graph $\mathcal{S}^{H}$ by removing the edges of index $e$ for which the coefficient $P_{\mathrm{ie}}^{\text {edg }}$ is enforced to vanish. Let us denote that $P_{i}^{\text {edg }}$ is the row vector defined from the components of the $i$ th row of $P^{\text {edg }}$ by extracting the components with edge indices in $\mathcal{S}^{\mathrm{H}, \mathrm{i}}$, and $\left(G^{h} P^{\text {nod }}\right)_{i}$ is the row vector defined from the components of the $i$ th row of $\left(G^{h} P^{\text {nod }}\right)$ by extracting the components with node indices in $\mathcal{S}^{\mathrm{H}, \mathrm{i}}$.

Then, it is proved in [10] that a necessary and sufficient condition of existence of a solution $P^{\text {edg }}$ to (7), for any $P^{\text {nod }}$, is the connectivity of subgraphs $\mathcal{S}^{\mathrm{H}, \mathrm{i}}$. It is also shown that to find a solution $P^{\text {edg }}$ satisfying (7) is equivalent to solve the flow problems

$$
P_{i}^{\mathrm{edg}} G^{\mathrm{H}, \mathrm{i}}=\left(G^{h} P^{\mathrm{nod}}\right)_{i} .
$$

The solution of such a linear system can be written as

$$
P_{i}^{\text {edg }}=\left(P_{i}^{\text {edg }}\right)^{\prime}+\left(P_{i}^{\text {edg }}\right)^{\prime \prime}
$$

where in a graph context

- the term $\left(P_{i}^{\text {edg }}\right)^{\prime}$ is a particular solution of the flow problem, which can be computed from a spanning tree of the subgraph $\mathcal{S}^{\mathrm{H}, \mathrm{i}}$;

- the transposed of $\left(P_{i}^{\text {edg }}\right)^{\prime \prime}$ belongs to the kernel of $\left(G^{\mathrm{H}, \mathrm{i}}\right)^{T}$, which can be defined from a set of independent cycles of the subgraph $\mathcal{S}^{\mathrm{H}, \mathrm{i}}$.

Thus, the degrees of freedom for the minimization phase are the components in the kernel of $\left(G^{\mathrm{H}, \mathrm{i}}\right)^{T}$ for each $i$, for which we can easily build bases.

We also observe that, whatever the matrix $P^{\text {nod }}$ is, a matrix $P^{\text {edg }}$ satisfying (7) can be built from the rows $\left(P_{i}^{\text {edg }}\right)^{\prime}$; it can be 
used as prolongation matrix, but the energy-minimization property is not satisfied.

\section{B. From the Optimization Problem to a Linear System}

Introducing appropriate numbering and projection operators for the support constraints, problem (8) is reduced to the resolution of the linear system

$$
\left(B^{T} D B\right) \Gamma=-B^{T} D \tilde{P}^{\text {edg }}
$$

where we have gathered information from all the subgraphs.

- The solution $\Gamma$ is the vector whose components give the coefficients of $\left(\left(P_{i}^{\text {edg }}\right)^{\prime \prime}\right)^{T}$ in the bases of the kernel of $\left(G^{\mathrm{H}, \mathrm{i}}\right)^{T}$.

- The matrix $B$ gathers the basis vectors of these different kernels. It is a sparse full-rank matrix which is assembled during the resolution of flow problems (9).

- The matrix $D$ is block diagonal and its diagonal blocks are the matrices $K_{e}$ involved in (8).

- The vector $\tilde{P}^{\text {edg }}$ gathers the particular solutions $\left(P_{i}^{\text {edg }}\right)^{\prime}$ from all flow problems (9).

\section{Properties of System (11)}

The matrices $K_{e}$ being symmetric positive definite (SPD), the matrix $B^{T} D B$ is $\mathrm{SPD}$, and we can use the conjugate gradient (CG) to solve the system. In most cases, the matrix is not assembled; we have only to compute matrix-vector products, i.e., operations with $B, D$, and $B^{T}$.

For evaluating the behavior of the CG method on system (11), a rough estimate of the conditioning number of the matrix is given by the inequality

$$
\operatorname{cond}_{2}\left(B^{T} D B\right) \leq \operatorname{cond}_{2}\left(B^{T} B\right) \operatorname{cond}_{2}(D)
$$

where cond denotes the conditioning number relative to the 2-norm.

The matrix $B^{T} B$ is similar to a block-diagonal matrix whose blocks are of the form $\left(\tilde{B}^{\mathrm{H}, \mathrm{i}}\right)^{T} \tilde{B}^{\mathrm{H}, \mathrm{i}}$ where $\tilde{B}^{\mathrm{H}, \mathrm{i}}$ gathers the basis of the kernel of $\left(G^{\mathrm{H}, \mathrm{i}}\right)^{T}$. Therefore, the conditioning of $B^{T} B$ remains low and independent of the global dimension of the problem.

Depending on the choice of the matrices $K_{e}$, the conditioning of $D$ may be slightly dependent of the global dimension.

Anyway, observe that the system (11) has not to be solved accurately because its solution is only needed to improve the convergence of iterative methods for the initial system (2).

\section{NUMERICAL EXAMPLES AND COMMENTS}

\section{A. Description of Test Problems}

A 2-D eddy current problem in a L-shape conducting domain $\Omega$ is solved (Fig. 1). An $\mathbf{E}$-field formulation is used where an exterior magnetic field $\mathbf{H}$ is imposed on the domain boundary. An implicit Euler scheme with time parameter $\Delta t$ is used for time discretization. A problem similar to (1) with the coefficients $\delta=\Delta t / \mu$ and $\alpha=\sigma$ has to be solved at each time step. The source term is given by the electric field $\mathbf{E}$ at the previous instant and $\partial \mathbf{H}_{z} / \partial t$ on the exterior boundary at the current instant.

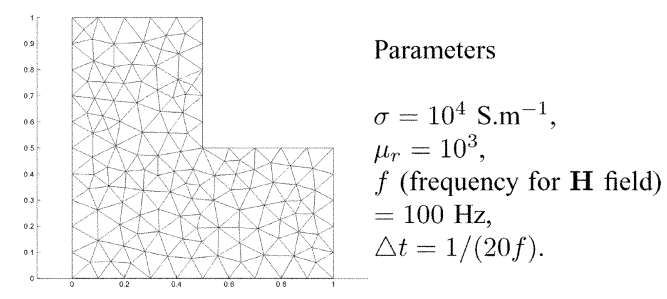

Fig. 1. Domain $\Omega$ and parameters of the problem.

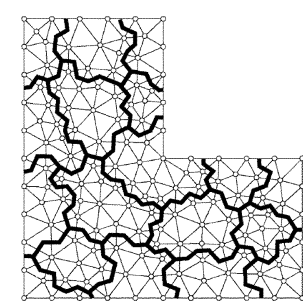

(a)

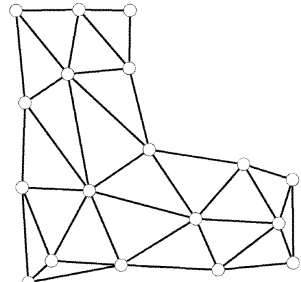

(b)
Fig. 2. Fine graph with partition in bold lines and coarse graph. (a) Initial graph and partition in bold lines. (b) Representation of the coarse graph related to 2(a).

In order to evaluate the efficiency according to the increase of the number of degrees of freedom (DOFs), different meshes are used with a decreasing maximal diameter $h_{\max }$ of the mesh elements.

As Reitzinger and Schöberl do in [5], we introduce a partition of the fine nodes:

$$
\left\{1, \ldots, N^{h}\right\}=\bigcup_{n=1}^{N^{H}} H_{n} .
$$

Fig. 2(a) illustrates an automatically determined partition where node aggregates are separated by bold lines. This partition induces a decomposition of domain $\Omega$ into overlapping subdomains $\tilde{\Omega}_{n}$ defined by

$$
\tilde{\Omega}_{n}=\bigcup_{p \in H_{n}} \operatorname{supp}\left(\phi_{p}^{h}\right) .
$$

The graph $\mathcal{S}^{H}$ is constructed as follows: an edge of extremities $n$ and $m$ is introduced if, and only if, $\tilde{\Omega}_{n}$ and $\tilde{\Omega}_{m}$ intersects. The coarse graph corresponding to the partition of Fig. 2(a) is represented in Fig. 2(b).

Each subdomain $\tilde{\Omega}_{n}$ is extended to all the nearest nodes in order to define the domain $\Omega_{n}$ involved in the definition of the support of the coarse nodal function $\phi_{n}^{H}$. Without such an extension, no degree of freedom would be available for the minimization problem and our method would coincide with the Reitzinger and Schöberl method (RS method).

For the 3-D eddy current problem, the formulation and the coefficients used are analogous to the 2-D case; the only difference is the chosen domain: the unit cube.

\section{B. Results}

The critical part of the computation is the construction of the coarse basis from the initial mesh. Results with decreasing $h_{\max }$ are given in Table I for the 2-D case and Table II for 3-D case. The number of unknows for system (11) is comparable to the number of DOFs in the 2-D case; it can be between twice and 
TABLE I

RESOLUTION OF SySTEM (11), 2-D CASE

\begin{tabular}{ccccc}
\hline \hline & $h_{\max }=0.2$ & 0.1 & 0.05 & 0.025 \\
\hline Number of DOFs & 119 & 401 & 1522 & 6143 \\
Number of unknowns in (11) & 84 & 226 & 1143 & 4670 \\
CG iterations $(A$ or $S$ ) & 5 & 6 & 7 & 9 \\
CG iterations (Id) & 2 & 2 & 2 & 3 \\
\hline \hline
\end{tabular}

TABLE II

RESOlution OF SySTEM (11), 3-D CASE

\begin{tabular}{ccccc}
\hline \hline & $h_{\max }=0.4$ & 0.2 & 0.1 & 0.05 \\
\hline Number of DOFs & 302 & 1559 & 11037 & 84403 \\
Number of unknowns (11) & 447 & 2910 & 25540 & 209087 \\
CG iterations $(A)$ & 4 & 8 & 13 & 26 \\
CG iterations (S) & 5 & 11 & 13 & 22 \\
CG iterations (Id) & 3 & 5 & 5 & 6 \\
\hline \hline
\end{tabular}

TABLE III

MEAN Number OF ITERATIONS AFTER 20 Time StePs, 2-D CASE

\begin{tabular}{ccccc}
\hline \hline solver & $h_{\max }=0.2$ & 0.1 & 0.05 & 0.025 \\
\hline SSOR & 26 & 36 & 67 & 135 \\
RS method & $4(2)$ & $7(2)$ & $11.5(3)$ & $18.5(4)$ \\
without min. & $4(2)$ & $6(2)$ & $10(3)$ & $16.5(3)$ \\
Id & $4(2)$ & $6(2)$ & $10(3)$ & $13(3)$ \\
$S$ & $4(2)$ & $6(2)$ & $9(3)$ & $11(3)$ \\
$A$ & $4(2)$ & $6(2)$ & $9(3)$ & $11(3)$ \\
\hline \hline
\end{tabular}

TABLE IV

NUMBER OF ITERATIONS FOR THE FIRST TIME STEP, 3-D CASE

\begin{tabular}{ccccc}
\hline \hline solver & $h_{\max }=0.4$ & 0.2 & 0.1 & 0.05 \\
\hline SSOR & 50 & 63 & 113 & 173 \\
RS method & $3(2)$ & $5(2)$ & $8(2)$ & $14(3)$ \\
without min. & $3(2)$ & $6(2)$ & $8(2)$ & $14(3)$ \\
Id & $3(2)$ & $5(2)$ & $8(2)$ & $14(3)$ \\
$S$ & $3(2)$ & $5(2)$ & $8(2)$ & $14(3)$ \\
$A$ & $3(2)$ & $5(2)$ & $7(2)$ & $13(3)$ \\
\hline \hline
\end{tabular}

three times the number of DOFs in the 3-D case. For the matrices $K_{e}$, we test several choices:

- matrices extracted from the global matrix $A$ of the problem denoted by $A$ in Tables I-IV;

- matrices extracted from the matrix defined from $\int_{\Omega} \delta \operatorname{curl} E \cdot \operatorname{curl} E^{\prime}$ and a local regularization denoted by $S$ in Tables I-IV;

- matrices are all equal to the identity, i.e., $D$ is also the identity in (11), denoted by Id.

The number of nonpreconditioned CG iterations needed to divide by 10 the norm of the residual, when computing the minimization system (11), is almost independent of the mesh size in the 2-D case; in the 3-D case, it remains true for choice Id, but not for choice $A$.

The CG with various preconditioners is used for computing the solutions at each time:

- the classical SSOR method;

- the RS method;

- our multilevel method for the Id, $A$, and $S$ cases and also for the case denoted "without min" where we only use the prolongation matrix built from the rows $\left(P_{i}^{\mathrm{edg}}\right)^{\prime}$.
For multilevel preconditioner, we use a $\mathrm{V}(1,1)$-cycle [1] with the smoother proposed in [3]. The number of levels is given in brackets when applicable. The computation stops when the norm of the residual is divided by $10^{10}$, the initial value is the solution for the previous time step. The mean number of iterations for this computation is favorable for our method as shown in Table III for the 2-D case. For the 3-D case, we only give the number of iterations for the first step in Table IV; the interest of our method is less obvious in this case, and the cost of solving (11) is not justified.

Observe that our method requires extra work to obtain the coarse basis compared to the RS method. Nevertheless, for timedomain computation, this initial effort is justified in the 2-D example, but not in the 3-D example.

\section{CONCLUSION}

We propose an algebraic multilevel method for linear systems coming from incomplete first-order edge element discretization. Many parameters in this generic presentation can be tuned in view of specific applications. For instance, for the time-harmonic problem with $\left|\alpha h_{\max }^{2}\right| \ll 1$, the method denoted by $S$ or by Id can be used. In order to balance the computational work between the construction of the coarse basis and the resolution of the initial system, the number of unknowns in problem (11) can also be decreased by removing some columns in $B$. Nonetheless, constraint (7) and, therefore, compatibility relation (3) is always ensured. Finally, an efficient implementation has to be implemented in order to test the methods on realistic examples.

\section{REFERENCES}

[1] U. Trottenberg, C. W. Oosterlee, and A. Schüller, Multigrid. San Diego, CA: Academic, 2001. with contributions by A. Brandt, P. Oswald and K. St'uben.

[2] R. Hiptmair, "Multigrid method for Maxwell's equations," SIAM J. Numer. Anal., vol. 36, pp. 204-225, 1999.

[3] D. N. Arnold, R. S. Falk, and R. Winther, "Multigrid in $\mathbb{H}($ div $)$ and $\mathbb{H}($ curl)," Numer. Math., vol. 85, pp. 197-217, 2000.

[4] R. Hiptmair, "Finite elements in computational electromagnetism," Acta Numer, vol. 11, pp. 237-339, 2002.

[5] S. Reitzinger and J. Schöberl, "An Algebraic multigrid method for finite element discretizations with edge elements," Numer. Linear Algebra Appl., vol. 9, pp. 223-238, 2002.

[6] P. Bochev et al., "An improved algebraic multigrid method for solving Maxwell's equations," SIAM J. Sci. Comput., vol. 25, no. 2, pp. 623-642, 2003. electronic.

[7] P. Bochev et al., "Toward an h-independent algebraic multigrid method for Maxwell's equations," SIAM J. Sci. Comput., to be published.

[8] W. L. Wan, T. F. Chan, and B. Smith, "An energy-minimizing interpolation for robust multigrid methods," SIAM J. Sci. Comput., vol. 21, no. 4, pp. 1632-1649, 1999/00. electronic.

[9] P. Vanek, J. Mandel, and M. Brezina, "Algebraic Multigrid by Smoothed Aggregation for Second and Fourth Order Elliptic Problems,", citeseer.ist.psu.edu/vanek96algebraic.html, vol. 1, Tech. Rep. UCD-CCM-036, 1995. [Online]. Available.

[10] F. Musy, L. Nicolas, and R. Perrussel, "Gradient-prolongation commutativity and graph theory," C. R. Acad. Sci. Paris, vol. 341, no. 11, pp. 707-712, 2005.

Manuscript received June 25, 2005; revised November 21, 2005 (e-mail: ronan.perrussel@ec-lyon.fr). 